\title{
Therapeutic Equivalence: A Connecting Link Between Test Drug and Reference Drug
}

\section{R. Santosh Kumar and G.V.R.L. Soujanya}

GITAM Institute of Pharmacy, GITAM (Deemed To Be University) Rushikonda, Visakhapatnam-530045, Andhra Pradesh, India.

\section{ABSTRACT}

The concept of therapeutic equivalence is becoming increasingly important in today's cost - conscious environment. Though an effective therapy already exists, but clinically equivalent therapy also becoming important. An improved toxicity profile better effects on symptoms and ease of administration may be important considerations. In these positive controls substantial effect is required to define equivalence. The goal is to prove that the new treatment is not inferior to standard, since providing that two treatments are equal is not possible. The superiority trials demonstrate the better efficacy of the treatment against the concurrent placebo control. Innovative drugs become available for the treatment of number of diseases. These, new products may offer some specific advantages over the standard drugs. The Placebo controlled trials are invariably unethical, when known effective therapy is available for the condition being studies. The active controlled trials are used extensively in the development of new pharmaceuticals. The equivalence limit is defined by a lower equivalence or upper equivalence limit. These, principles are proposed for setting such limits, depending on the objective of the study placebo conditions and methods based on statistical properties.
\end{abstract}

Keywords: Therapeutic equivalence, Test Drug, Reference Drug, Placebo

Article Info: Received 10 June 2019; $\quad$ Review Completed 18 August 2019; $\quad$ Accepted 25 August 2019; $\quad$ Available online 30 August 2019

\section{Cite this article as:}

Santosh Kumar R, Soujanya G.V.R.L., Therapeutic Equivalence: A Connecting Link Between Test Drug and Reference Drug, Journal of Drug Delivery and Therapeutics. 2019; 9(4-s):829-831 http://dx.doi.org/10.22270/jddt.v9i4-s.3395

\section{*Address for Correspondence:}

R. Santosh Kumar, GITAM Institute of Pharmacy, GITAM (Deemed to be University), Gandhinagar, Visakhapatnam530045, Andhra Pradesh, INDIA.

\section{INTRODUCTION}

In pharmaceutical industries, for the development of therapeutic entities. Phase II and III of clinical trials are conducted for proving the effectiveness and safety of the drug. The goal of the trials is to obtain better efficacy of treatment against concurrent placebo control trial. For the treatment of number of diseases the remodelled or innovative drugs are used. A new therapeutic modalities are developed to compete with the standard products on the market. Therapeutic modalities may have some specific advantages over the standard drug. It has better safety profile improvement on the quality of life, an easy administration route, short duration of treatment and mainly the reduction of cost. Hence the new drug contains more benefits. But it is proving that its effectiveness is more than that of the standard drug. The sponsors should require the evidence that the test product is efficient than the standard. More efficacy and with some specific added values with safety profile provided to the new treatment is called Therapeutic equivalence. Hence these studies of therapeutic equivalence are called Non inferiority trials. Therapeutic equivalence only requires obtaining the efficacy of new treatment than the standard so it is referred as one sided equivalence. The patent of an innovative product expires, other companies may manufacture generic copies under drug price competition and patient term restoration act passed in 1984, Through the Abbreviated New drug Application (ANDA). The generic copies must be equivalent to the rearranged product based on pharmacokinetic pk such as area under plasma concentration time curve, area under curve (AUC) or peak concentration (cmax) obtained from bioequivalence studies. The average bioavailability of generic product is neither too high nor too low as compared to that of reference product. Hence, bioequivalence has importance in two sided equivalence. It has average bioequivalence, population bioequivalence and individual bioequivalence that are described in equivalence trials.

\section{ONE SIDED EQUIVALENCE AND NON INFERIORITY TRIALS}

The average efficacy of test and standard value of the drug would be $\mu \mathrm{T}$ and $\mu \mathrm{S}$. large value gives a better efficacy. For an advanced trial, investigators are interested in detecting 
whether a difference in efficacy exists between the test and standard drugs. Hence this question can be given by following formulation of Hypothesis.

$$
\text { Ho: } \mu \mathrm{T}=\mu \mathrm{S} \text { versus Ha: } \mu \mathrm{T} \neq \mu \mathrm{S}
$$

Equation -1 is in the form of two sided hypothesis. All other argued that the advanced trial should be formulated as one sided.

$$
\mu \mathrm{a}: \mu \mathrm{T}>\mu \mathrm{S} \quad-2
$$

After the null hypothesis test there is no difference in both the equations. It can be proven at significant level. The null hypothesis $\mu \mathrm{T}-\mu \mathrm{S}$ is not rejected the concentration of trial should never be proven in or trial. The difference may result from a poorly conducted trial that provides variable data and a large experimental error. Another side well designed and carefully executed trials generate reliable data to provide estimates of treatment effects with high accuracy and precision. The paradox is that the form of hypothesis in equation 1 is wrong hypothesis for obtaining of one sided equivalence in a non inferiority trial.

The correct hypothesis will be one sided hypothesis to test whether test drug is more effect as standard drug.

$$
\text { Ho: } \mu \mathrm{T}-\mu \mathrm{S} \leq \mathrm{L} \text { versus Ha: } \mu \mathrm{T}-\mu \mathrm{S}>\mathrm{L} \quad-3
$$

$\mathrm{L}=$ maximum allowable limit of no clinical significance.

This trial objective is that the test drug is more effective than the standard drug. It follows the equation -3 for the continuous end points from a two group parallel design which is compared to test drug of nT patient to the $\mathrm{n} S$ standard drug. YT -YS are the observed means of test and standard drug, $S^{2}$ is poored variance.

$$
\mathrm{T}=\left(\mathrm{Ý}_{\mathrm{T}}-\mathrm{Y} \mathrm{S}-/ \mathrm{L}\right) / \mathrm{S}^{\wedge} 1 / \mathrm{nt}+1 / \mathrm{ns}>\mathrm{Z}(\alpha)
$$

$\mathrm{Z}(\alpha)$ is the $\alpha$ th upper quantile of the standard normal distribution equivalence is not only a testing procedure but also an estimation problem. The use of confidence interval for evaluation of both types of equivalence for one sided - (1 $-\alpha) 100 \%$ confidence limit is larger than lower equivalence limit.

$$
\text { (ÝT - ÝS })-Z(\alpha)\left(S^{\wedge} 1 / n t+1 / n s\right)
$$

The confidence interval approach is more than the testing hypothesis procedure. It provides magnitude and range of average difference between two treatments. For a two group parallel design with equal allocation. The same size per group required to give $(1-\beta)$. Hypothesis 3 can be estimated by following for

$$
\left.\mathrm{n}=2[\urcorner^{2} /\left(\infty+\mathrm{L}^{2}\right)\right][\mathrm{Z}(\alpha)+\mathrm{Z}(\beta)]^{2}
$$

Where $\infty=\mu \mathrm{T}-\mu \mathrm{S}>\mathrm{L}$ is assumed as true difference in average effect between test and standard drugs. $\neg^{2}$ is common variable. $Z(\beta)$ is the upper quantile of the standard normal distribution. From equation $6 \mathrm{Th}$ sample size is a decreasing function of true unknown difference $\mu \mathrm{T}-\mu \mathrm{S}$ when $\infty=0$ formula 6 reduce to.

$$
\left.\mathrm{n}=2[\urcorner / \mathrm{L}^{2}\right][\mathrm{Z}(\alpha)+\mathrm{Z}(\beta)]^{2}
$$

For the evaluation of one sided equivalence based on binary end points from a two group parallel trial. The corresponding hypothesis are given

$$
\text { Ho: } \mathrm{PT}-\mathrm{PS} \leq \mathrm{L} \text { vs. Ha: PT - PS > L } \quad-7
$$

Where PT and PS are the response rates of test and standard treatment equation -7 is rejected and the test drug is more effective than the standard drug.

$$
\mathrm{Z}=(\mathrm{PT}-\mathrm{PS}-\mathrm{L}) / \mathrm{SE}>\mathrm{Z}(\alpha)
$$

Where SE ${ }^{2}=[\mathrm{pr}(1-\mathrm{pr}) / \mathrm{nT}]+[\mathrm{PS}(1-\mathrm{PS} / \mathrm{ns})]$. The corresponding lower $(1-\alpha) 100 \%$ confidence limit and sample size estimation formula.

$$
\begin{aligned}
& (\mathrm{PT}-\mathrm{PS})-\mathrm{Z}(\alpha) \mathrm{SE} \text { and } \\
\mathrm{n} & =\left\{\mathrm{SE}^{2} /[(\mathrm{PT}-\mathrm{PS})+\mathrm{L}]^{2}\right\}\{\mathrm{Z}(\alpha)+\mathrm{Z}(\beta)\}^{2} .
\end{aligned}
$$

This is the assessment of equivalence between 2 survival functions.

\section{ACTIVE CONTROL EQUIVALENCE TRIALS}

The non inferiority trials demonstrated that the efficacy of test drug is more than the standard drug they are also referred as Active control equivalence trials. The critical criterion is the effectiveness of the standard drug. The test and standard drug are therapeutic equivalent that they can be either efficacious or inefficacious. A series of 6 trials were conducted to find out the effectiveness of new drug. Ex: Imipramine.

(HAM - D) from baseline between test drug and imipramine both drug produce a clinically meaningful mean reduction in HAM - D. HAM- D is not rejected at $5 \%$ of significance level. The power to detect a $30 \%$ difference is quite low.HAM - D and imipramine is compared with the placebo. Both the test drugs and imipramine are ineffective as compared to the placebo. If a concurrent placebo control were not there in 6 trials, the test drug would have been stated the effect based on conjecture that the test drug and imipramine have similar efficacy. This example explains about the concurrent placebo control in active control equivalence trials unless the active standard has been proven efficacious inadequate well controlled studies. ACET has 3 treatments, a test drug active standard and placebo concurrent control. In addition to evaluate of therapeutic equivalence between test and standard. Hence the following hypothesis is formulated to evaluate these 2 major objectives.

$$
\begin{aligned}
& \text { Ho: } \mu \mathrm{T}-\mu \mathrm{S} \leq \mathrm{L} \text { or } \mu \mathrm{T}-\mu \mathrm{P} \leq \mathrm{O} \text { or } \mu \mathrm{S}-\mu \mathrm{P} \leq 0 \text {. Vs. } \\
& \text { Ha: } \mu \mathrm{T}-\mu \mathrm{S}>\mathrm{L} \text { and } \mu \mathrm{T}-\mu \mathrm{P}>0 \text { and } \mu \mathrm{S}-\mu \mathrm{P}>0
\end{aligned}
$$

Where $\mu \mathrm{p}$ is average efficacy of placebo. There are two more one sided hypothesis for evaluation of effectiveness in test and standard drugs as compared to placebo.

$$
\begin{aligned}
& \text { Ho } \mathrm{T}: \mu \mathrm{T}-\mu \mathrm{P} \leq 0 \text { Vs } \mu \mathrm{T}-\mu \mathrm{P}>0 \text { and } \\
& \text { Ho } \mathrm{S}: \mu \mathrm{S}-\mu \mathrm{P} \leq 0 \text { Vs } \mu \mathrm{S}-\mu \mathrm{P}>0
\end{aligned}
$$

Parameter space of null hypothesis in equation 9 is union of spaces of 3 null hypotheses in equation 3 and 10. Parameter space of alternative hypothesis is the intersection of spaces. Hence, all 3 one sided null hypothesis are rejected at significant level by intersection union principle. The null hypothesis in equation 9 is rejected at $\alpha$ significance level and it is concluded that both test and reference drugs are superior to placebo and are equivalent. This procedure is called as 3 one sided test procedures. This procedure can control consumes risk under the nominal level of significance.

\section{EQUIVALENCE LIMIT}

For some bioequivalence testing and some therapeutic areas the equivalence limits are usually expressed in terms of standard response. Equivalence limit are unknown parameters. For one sided therapeutic equivalence the lower limit L is determined by previous experience about estimated relative efficacy along with placebo and by maximum allowance below which clinicians consider to be therapeutic acceptable. It is not good to have an equivalence limit for evaluation of more efficacy as large as or even 
larger than the treatment difference between the standard drug and the placebo. Ex: 3.2 limits are selected in treatment of perennial allergic rhinitis. It is selected for evaluation of therapeutic equivalence between a test and standard drug because 3.2 represents $25 \%$ of the sample range for the total symptom scores 3.2 limit not suitable for individual symptom scores 3.2 is for that total symptom scores 0.5 is for individual scores.

Another example is continuous infusion versus double bolus administration of alteplase (COBALT). For fibrinolytic therapy of suspected acute myocardial infection, the 30 day mortality rate is about $12 \%$ for placebo group and $8 \%$ for tissue plasminogen activator. Hence the treatment effect of tissue plasminogen activator against placebo is $4 \%$. Cobalt investigators employed an equivalence limit of $0.4 \%$. This limit is 1 / 10 of estimated relative treatment effect against placebo. This explains about the upper limit of deaths allowed for the double bolus alteplase to be considered Therapeutic equivalence to accelerated infusion is 4 more deaths per 1000 patients. This number is few than 5.0 per 1000 patients between alteplase and streptokinase. Therapeutic equivalence accelerated in a non inferiority trial should always be selected as a quantity smaller than the difference between standard drug and placebo that a superior trial is designed to detect. The ultimate and real goal of any inferiority trial is to prove that efficacy of the test treatment is better than that of placebo.

\section{RECENT DEVELOPMENT}

In the framework of hypothesis testing and regulatory considerations, kothmann et al have addressed the design and analysis of non inferiority trial based on survival for oncology trials. The European committee for medical products for human use (CHMP) issued a guideline for selection of non inferiority margin. Non inferiority testing has been applied to evaluation of accuracy of diagnostic devices.

\section{CONCLUSION}

For a non inferiority trial, the objectives of evaluation for equivalence between the test and the standard drugs and for superiority of both drugs over placebo should be clearly stated in the protocol. In addition, the equivalence limit should also be prespecified in the protocol with clinical and statistical justification. As indicated in $(\mathrm{CH})$ an adequate active control is non inferiority trial should be a widely used standard. Therapeutic equivalence should have more efficiency and standard safety profile of drug. In general the intention to treat (HT) analysis will provide an estimate for treatment effect of a smaller magnitude than the per protocol (PP) analysis. Hence, the IIT analysis is not necessarily more likely to conclude therapeutic equivalence.

\section{REFERENCES}

1. Chow, SC, L w, J.P. Design and analysis of bioavailability and bioequivalence studies. 3rd ED; CRC/ Chapman \& Hall: New York, NY, 2008.

2. The FDA guidance. Bioavailability and bioequivalence studies for orally administered drug Products - general considerations; food and drug administration Rockville, MD, 2003

3. Fisher, LD, The use of one sided tests in drug trials: An FDA advisory committee member's perspective. J. Biopharm. Stat. 1991, I, 151 - 156

4. Koch, G.C. One sided and two sided tests and p values. J. Biopharm Stat. 1991, I. 161 - 170.

5. Dubey, S.D. Some thoughts on the one sided and two sided tests. J.Biopharm Stat 1991, I. 139- 150.

6. Chow, S.C.; Liu, J.P Design and analysis of clinical trials concepts and methodologies, 2 nd ed. John wiley and sons: New York, NY, 2004.

7. Durrleman, S; Simon, R .planning and monitoring of equivalence studies. Biometrics 1990, 46, 329 - 336

8. Black welder, W.C. "Prove the null hypothesis "in clinical trials. Control. Clin. Trials 1982, 3, 345-353.

9. Ware, J.H.; Antman, C.G. equivalence trials .n.Engl, J.MED; 1997 $337,1159-1162$.

10. Makuch, R.W.; Simon, Resample size requirements for evaluating a conservative therapy, cancer treat. Rep. 1978, 6, 1037 - 1040.

11. Makuch, R.W. Johnson, M. Issues in planning and interpreting active control equivalence studies. J. Clin. Epidemiol. 1989, 42, $503-511$.

12. Liu, J.P .Letter to the editor on "sample size for therapeutic equivalence based on confidence interval" by S.C.Lin.Drug inf. J. 1995, 29, 1063 - 1064.

13. Wellek, S.A log -rank test for equivalence of two survivor functions. Biometrics. 1993, 49, 31-33.

14. Leber, P.D.Hazards of inference; the active control interpretation .Epilepsia 1989, 30,557 - 663.

15. Senn, S.Inherent difficulties with active control equivalences studies. Stat. Med. 1993, 12, 2367 - 2375.

16. Siegel, J.P. Equivalence and non inferiority .AM. Heart J. 2000,139, 8166 - 6170.

17. Berger, R.L.Multiparametric hypothesis testing and acceptance sampling .techno metrics. 1982. 24,295 - 300.

18. Hung, H.M.J. Noninferiority: A dangerous toy. ICSA Bull Jan. 2001, $27-29$.

19. Wittes, J.Active control trials: A Linguistic problem. ICSA Bull Jan $.2001,39-40$.

20. Simon, R. Bayesian design and analysis of active control clinical trials . Biometrics 1999, 55,484- 487.

21. Rothmann,M .; Li,N; Chen,G.; Chi,G.Y.H.;Thou, H.H. Design and analysis of non inferiority mortality in oncology Stat. Med. 2003, $22,239-264$

22. Ng, T.H.Non inferiority hypothesis and choice of non inferiority margin. Stat. Med.2009, 28, 1668 - 1679.

23. Lewis, J.A.; Mathin, D. Intention to treat - Who should use IIT? Br. J. Cancer 1993, 68, $647-650$. 